\title{
Hybrid Genetic Algorithm and Support Vector Regression Performance in CNY Exchange Rate Prediction
}

\author{
Feng Jiang \\ School of Statistics and Mathematics \\ Zhongnan University of Economics and Law \\ Wuhan, 430073 China \\ jeff20@163.com
}

\author{
Wenjun $\mathrm{Wu}$ \\ School of Statistics and Mathematics \\ Zhongnan University of Economics and Law \\ Wuhan, 430073 China \\ wenjoriewu@163.com
}

\begin{abstract}
In this paper, we predict CNY exchange rates in terms of hybrid genetic algorithm and support vector regression with a range of kernel functions. A BP neural network model is benchmarked with the hybrid model and then the hybrid genetic algorithm and support vector regression model are used to examine the accuracy of CNY exchange rate prediction. The intuitive and statistical performances of the hybrid model with linear, radical basis, polynomial and sigmoid functions are presented and analyzed by using the exchange rate data of USD/CNY, EUR/CNY and CNY/JPY. The empirical results show that the hybrid model is effective for studying the CNY exchange rate prediction.
\end{abstract}

Keywords-genetic algorithm; support vector regression; exchange rate

\section{INTRODUCTION}

At present, the issue of the exchange rate prediction is one of the best important problems in economics and finance. For example, Rime et al [1] illustrates that exchange rate prediction is urgently required in the exchange rate management for both governors and investors. On one hand, predicting the evolution of exchange rate can help investors worldwide to make portfolio decisions. On the other hand, it plays a significant role for the governors to make macroeconomic policies. In academic field, the accurate and reliable forecasting of exchange rate is one of the most challenging tasks. Given the non-linear and non-stationary characters, more effective techniques are needed for their prediction. There have great efforts in recent years to improve the forecasting performance which starts from the conventional time series statistical methods. At the same time the machine learning technique has been used due to its high efficiency and accuracy. The machine learning methodologies include the artificial neural networks (ANNs), such as back propagation (BP) neural network, and the support vector machine (SVM) etc. SVM was originally proposed to solve problems of classification and pattern recognition. The introduction of loss function by Cortes and Vapnik [2] extended the SVM method to the non-linear regression technique, that is, support vector regression (SVR) technique. Furthermore, Kwon \& Moon [3] pointed out the SVR model distinguished itself for its global optimization solution rather than local minima, and it made a compromise between model complexity and accuracy.
The generalized comparison made by Abraham and Yeung [4], Härdle [5], Sapankevych and Sangar [6], Khandani [7], Ögüt [8] and Papadimitriou [9] confirmed that the SVR is a state-of-art technique in exchange rate forecasting. Lam [10] pointed out that machine learning methods regarded numeric data directly as input, which made it contain more information than classical methodologies. To explore performance of different techniques, Dunis and Williams [11] explored the performance of several models: NN, RW, ARMA, ARCH, and a Logit model using the dataset of EUR/USD exchange rate. Bissoondeeal [12] discussed a neural network model against three traditional models, which included random walk, autoregressive moving average (ARMA) and generalized autoregressive conditional heteroskedasticity (GARCH) models, and then studied forecast UK and US exchange rates. Önder [13] gave comparison between neural network and traditional smoothing time series methods, and concluded that artificial neural networks without assumptions about the probability distribution of input variables and residuals, distinguished themselves for their performance in out-of-sample forecasting.

The experiments on the improvement of SVR model can be divided into three parts: the selection of variable, the optimization of parameters and the selection of kernel function. Ince and Trafalis [14] combined the ARIMA with SVM in order to improve the prediction of the EUR exchange rate. Their experiments illustrated that the proposed technique had higher accuracy than the logit/probit models. Brandl [15] introduced genetic algorithms into SVR model for variable selection. The proposed model outperforms the ANNs, the OLS regression and the ARIMA model on monthly out-of-sample forecasting for the EUR/USD, the USD/JPY and the USD/GBP rates. Rubio [16] proposed a heuristic method for parameter selection in LS-SVM. The results given by Chen and Wang [17] showed that the prediction of tourist demand in China had better performance with genetically optimized parameters under epsilon kernel function. Recently, genetic algorithm is popular in the economics and finance, which can optimize the accuracy of prediction. Sermpinis et al [18] discussed EUR exchange rates with hybrid rolling genetical gorithms - support vector regression. Motivated by the above papers, in this paper we investigate the hybrid gene algorithm with four kernel functions and then predict the CNY exchange rates. 
The rest of this paper is organized as follows. Section 2 introduces the theoretical methodologies used in our empirical research. Section 3 provides the exposition of the data source, empirical results and discussion, where the intuitive and statistical performance is presented. Finally, a conclusion is given.

\section{METHODOLOGY}

\section{A. Supporting Vector Regression}

The main idea of support vector machine is to minimize the generalization error bounds which refer to the real risk of a machine learning procedure. Using the mathematical notation, there is a structural formula: $R(w) \leq \operatorname{Re} m p(w)+\phi(n / h)$ where $\operatorname{Re} m p(w)$ refers to the empirical risk in the classification of samples and $\phi(n / h)$ means the confidential risk representing the confidence level of a classifier.

The SVR model is a direct extension of the SVM. The basic idea of regression is to find a function whose deviation from the actual data locates in the predetermined scale. The support vector (SV) refers to the error tolerance boundary produced by a minimization procession. Supposing the function in linear form $f(x)=w^{T} x+b$, the detection procedure can be transferred to an optimization problem:

$$
\begin{array}{r}
\min \left(\frac{1}{2}\|w\|^{2}+C \sum_{i=1}^{n}\left(\zeta_{i}+\zeta_{j}\right)\right) \\
\text { subject to }\left\{\begin{array}{c}
y_{i}-\left(w x_{i}+b\right) \leq \varepsilon+\zeta_{i} \\
\left(w x_{i}+b\right)-y_{i} \leq \varepsilon+\zeta_{j} \\
\zeta_{i}, \zeta_{j} \geq 0
\end{array}\right.
\end{array}
$$

Here $\varepsilon$ is the error tolerance, $\zeta_{i}, \zeta_{j}$ are slack variables, and $w=\sum_{i=1}^{n}\left(\alpha_{i}-\alpha_{j}\right) x_{i}$ in which $\alpha_{i}, \alpha_{j}$ are Lagrange multipliers.

The method to solve nonlinear problem is to project the dataset to a higher-dimensional space by introducing a kernel function. In our research, we explore four kernel functions: linear, polynomial, radical basis function (RBF), and Sigmoid function. The mathematical form is presented as follows:

$$
\begin{aligned}
& \text { Linear: } K_{1}\left(x, x_{i}\right)=x^{T} x_{i} \\
& \text { Polynomial: } K_{2}\left(x, x_{i}\right)=\left(\gamma x^{T} x_{i}+r\right)^{d}, \gamma>0 \\
& \text { RBF: } K_{3}\left(x, x_{i}\right)=e^{-\gamma\left\|x-x_{i}\right\|^{2}}, \gamma>0 \\
& \text { Sigmoid: } K_{4}\left(x, x_{i}\right)=\tanh \left(\gamma x^{T} x_{i}+r\right)
\end{aligned}
$$

\section{B. Cross Validation-Grid Search}

Cross validation is a statistical analysis method to validate the property of classifier. The basic idea is to group the dataset into two parts: one is the train set, the other is the validation set. The process is to train the classifier using the train set and test the trained model by the validation set. In our exploration, we use the $\mathrm{K}$-fold Cross Validation (K-CV) technique combining the grid search (GS) method. The way is to divide the original data into $\mathrm{K}$ groups to avoid over-studying and lack-studying.
Using the CV-GS method, the best global solution is obtained by choosing the least parameter c \& g under the highest validation accuracy of classification. If the search scale is broad, however, it takes a long time to search for the best c \& g. Therefore, we consider the inspiration algorithm named genetic algorithm to shorten the parameter searching time.

\section{Genetic Algorithm}

The genetic algorithm origins from the computer simulation research of the biological systems. Inspired by the biological simulation technology, Holland from University of Michigan created this self-adaptive probable optimization methodology based on the genetic and evolutional mechanism in biology. Distinguished for its applicable, efficient and robust property, the GA algorithm has been applied in many optimization problems.

\section{RESULTS AND DISCUSSIONS}

\section{A. Data Source and Pretreatment}

The daily average data of exchange rates USD/CNY, EUR/CNY and CNY/JPY are all .obtained from the site http://fx.sauder.ubc.ca/ and Wind Database. The whole dataset is gathered from 1 January 2015 to 30 December 2015. And the total 250 values are divided into the trained set and test set, 195 of which are set as the trained set and 30 values are regarded as test set. Using the rolling prediction method, the exchange rate data in five days is set as input, and the following day is predicted as the output.

\section{B. Intuitive Performance}

After introducing all the techniques involved in the research, we choose one certain kernel function to observe the performance of various exchange rates. Radial basis function (RBF), the most commonly used kernel function in the existing research, is chosen to test the novel hybrid model in daily step using exchange rate data USD/CNY, EUR/CNY and JPY/CNY respectively. The forecasting results of $\mathrm{BP}$ neural network are plotted for daily exchange rate USD/CNY, EUR/CNY and JPY/CNY in Figs. 1 3. Especially, from Fig 1, we know that BP neural network prediction of daily exchange rate USD/CNY is obviously different from the real data. From Figs 2 and 3, the prediction of BP neural network for daily exchange rate EUR/CNY and JPY/CNY can be still accepted.

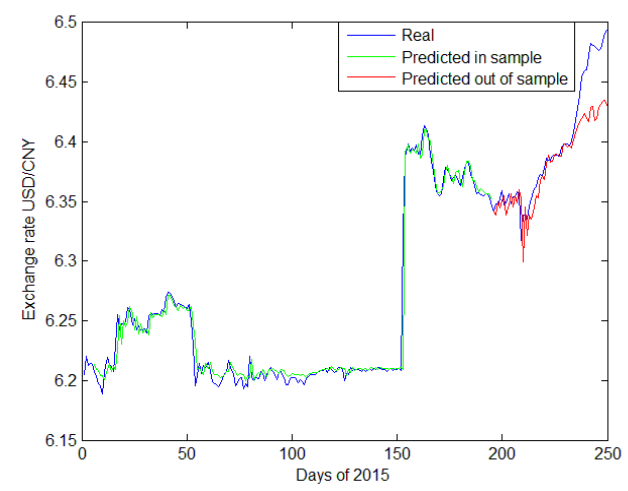

Fig. 1. BP neural network prediction of daily exchange rate USD/CNY 


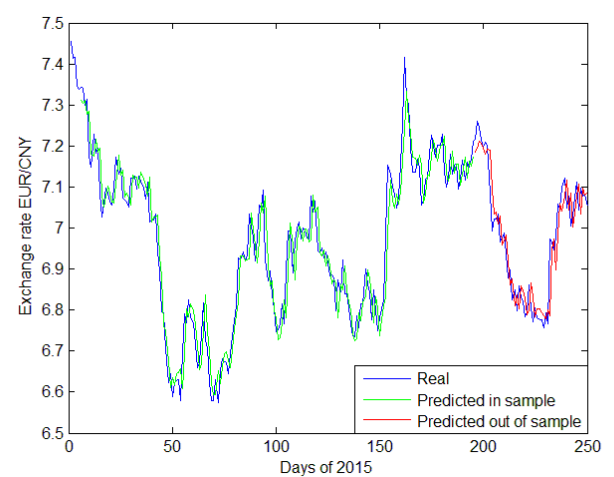

Fig. 2. BP neural network prediction of daily exchange rate EUR/CNY

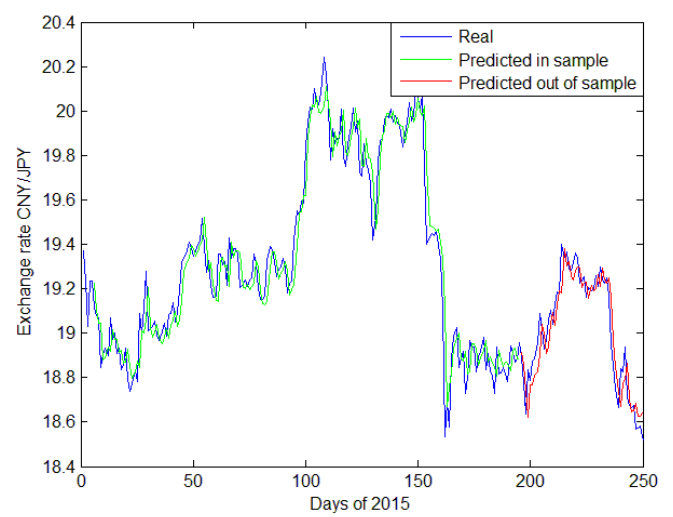

Fig. 3. BP neural network prediction of daily exchange rate CNY/JPY

In Figs $4 \sim 6$, the forecasting results of GA-SVR are plotted for daily exchange rate USD/CNY, EUR/CNY and JPY/CNY. From Figs. 1 and 4, we see that GA-SVR prediction of daily exchange rate USD/CNY is more effective than BP neural network. For daily exchange rate EUR/CNY and JPY/CNY, the forecasting results are still valid between BP neural network and GA-SVR method.

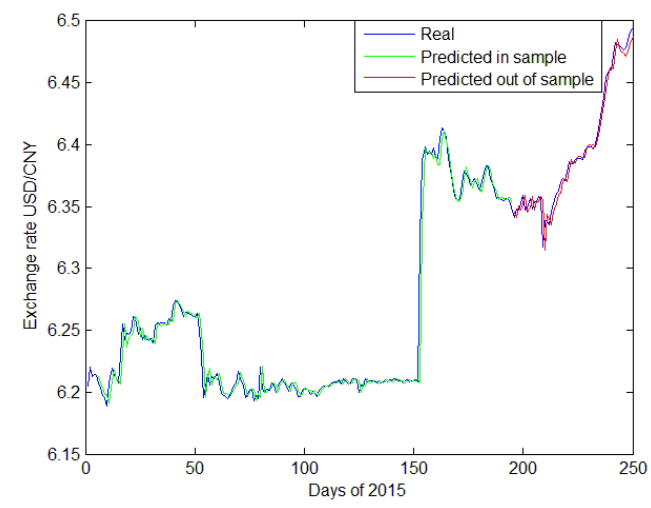

Fig. 4. GA-SVR prediction of daily exchange rate USD/CNY

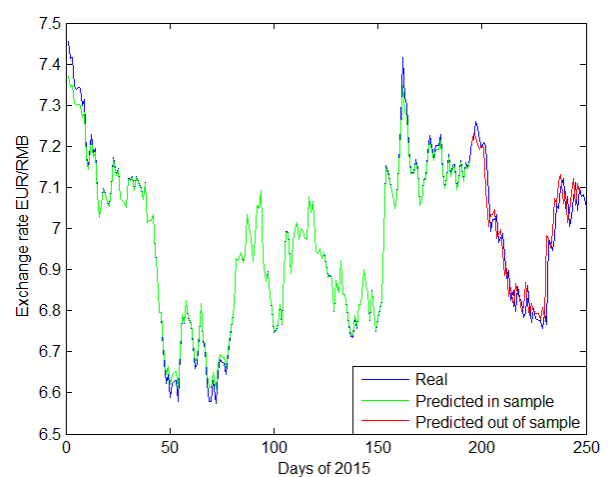

Fig. 5. GA-SVR prediction of daily exchange rate EUR/CNY

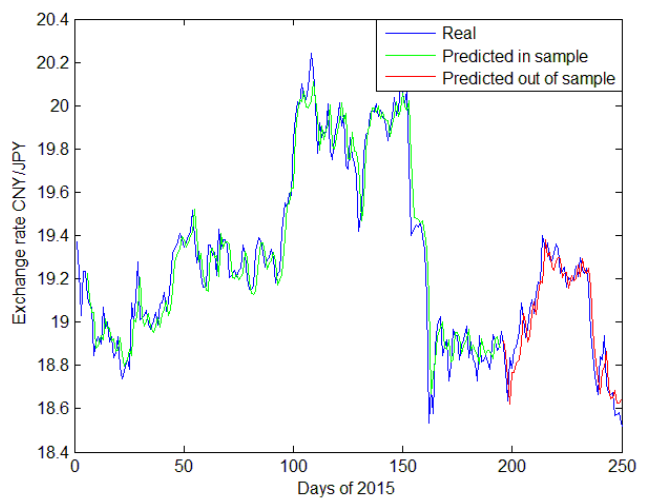

Fig. 6. GA-SVR prediction of daily exchange rate CNY/JPY

In terms of in-sample data and fluctuations caused by certain event, both BP neural network and GA-SVR have good prediction performance. As can be seen in the figures, the CNY exchange rates have substantial fluctuation during the whole year of 2015. Specifically, since the point of 150th day, there is a fast depreciation of CNY exchange rate and the volatility increases compared with the first two quarters. This change is probably due to the policy of exchange rate reform in China on the 11 August 2015.

As for out-of-sample data, however, GA-SVR outperforms the BP models. Comparing Fig. 2, Fig. 3 and Fig. 4 with other graphs, the GA-SVR not only catches the tendency of exchange rates, but the details of changes as well. For the prediction of USD/CNY, EUR/CNY and CNY/JPY exchange rate, the exact accuracy of both models is not apparent and is still needed to be explored. Therefore, in the next subsection, we would discuss the statistical results of more kernel functions.

\section{Statistical Performance}

With in-sample and out-of-sample data, the statistical performance of linear kernel (GA-L-SVR), polynomial kernel (GA-P-SVR), RBF (GA-R-SVR) and sigmoid function (GA$\mathrm{S}-\mathrm{SVR}$ ) is explored combined with genetic algorithm.

The USD/CNY, EUR/CNY and CNY/JPY exchange rate is classified according to the models, of which the Mean Squared Error (MSE) is presented in Table1 and Table 2. 
TABLE I. MSE OF GA-SVR IN SAMPLE

\begin{tabular}{|c|c|c|c|c|}
\hline \multirow{2}{*}{ MSE } & \multicolumn{4}{|c|}{ GA-SVR } \\
\cline { 2 - 5 } & $\boldsymbol{G A}-\boldsymbol{R}-\boldsymbol{S} \boldsymbol{V} \boldsymbol{R}$ & $\boldsymbol{G A}-\boldsymbol{L}-\boldsymbol{S} \boldsymbol{V R}$ & $\boldsymbol{G A}-\boldsymbol{S}-\boldsymbol{S} \boldsymbol{R}$ & $\boldsymbol{G A}-\boldsymbol{P}-\boldsymbol{S} \boldsymbol{V R}$ \\
\hline USD & 0.01019 & 0.01002 & 0.01098 & 0.11485 \\
\hline EUR & 0.01578 & 0.01746 & 113.40900 & 0.06753 \\
\hline JPY & 0.01578 & 0.01774 & 0.04912 & 0.06026 \\
\hline
\end{tabular}

TABLE II. MSE OF GA-SVR OUT OF SAMPLE

\begin{tabular}{|c|c|c|c|c|}
\hline \multirow{2}{*}{ MSE } & \multicolumn{4}{|c|}{ GA-SVR } \\
\cline { 2 - 5 } & $\boldsymbol{G A}-\boldsymbol{R}-\boldsymbol{S} \boldsymbol{V} \boldsymbol{R}$ & $\boldsymbol{G A}-\boldsymbol{L}-\boldsymbol{S} \boldsymbol{V} \boldsymbol{R}$ & $\boldsymbol{G A}-\boldsymbol{S}-\boldsymbol{S} \boldsymbol{V} \boldsymbol{R}$ & $\boldsymbol{G} \boldsymbol{A}-\boldsymbol{P}-\boldsymbol{S} \boldsymbol{V} \boldsymbol{R}$ \\
\hline USD & 0.00901 & 0.00974 & 0.00943 & 0.12622 \\
\hline EUR & 0.04630 & 0.04532 & 274.84700 & 0.10991 \\
\hline JPY & 0.04395 & 0.04506 & 0.13623 & 0.06026 \\
\hline
\end{tabular}

For USD/CNY prediction, the performance of GA-L-SVR in sample is the best while the accuracy of out of sample prediction with GA-R-SVR is the highest among four models. At the same time, GA-P-SVR has the largest error. The results indicate that linear kernel function is the most suitable for in sample prediction, but for out of sample forecasting, radical basis function (RBF) would be the best choice. No matter what sample it is, polynomial kernel function is the worst option.

To predict EUR/CNY, the figures show that in contrast of $\mathrm{USD} / \mathrm{CNY}$, the performance of RBF kernel function in sample has the most accurate prediction while linear kernel function performs best for out of sample data. Furthermore, the sigmoid kernel function, which would be the last choice for EUR/CNY prediction, has mighty error.

In the prediction of CNY/JPY, both in sample and out of sample errors demonstrate that RBF function has the highest accuracy. In addition, the polynomial one has the lowest accuracy for data in the sample while the sigmoid function is the worst choice to predict data out of sample.

In summary, the RBF kernel function and linear kernel function can compete for CNY exchange rate prediction, while sigmoid function and polynomial function would be a bad choice in common.

\section{CONCLUSION}

In the paper, the hybrid genetic algorithm and SVR with linear kernel, polynomial kernel, RBF and sigmoid function have been discussed. The hybrid model is used to predict daily CNY exchange rates. The statistical performances with in-sample and out-of-sample data show that the model is more effective than BP neural network. The results also show that the radical basis function (RBF) could be the best choice for the USD/CNY or CNY/JPY exchange rate data and the linear kernel function could be the most efficient one for the EUR/CNY exchange rate data. Based on the genetic algorithms, the study on the improvement of parameter selection technique could be developed in the future to improve the accuracy. Furthermore, robustness and sensitivity of the parameters can be discussed in the future.

\section{ACKNOWLEDGMENT}

The work is supported by the National Natural Science Foundation of China under Grant 61304067 and the Natural Science Foundation of Hubei Province of China under Grant 2013 CFB443.

\section{REFERENCES}

[1] D. Rime, L. Sarno, E. Sojli, "Exchange rate forecasting, order flow and macroeconomic information," J. Int. Econ., vol. 80, pp. 72-88, 2010.

[2] C. Cortes, V. Vapnik, "Support-vector networks," Machine Learning, vol. 20, pp. 273-297, 1995.

[3] Y.K. Kwon, B.R. Y.K. Moon, "A hybrid neurogenetic approach for stock forecasting," IEEE Trans. Neural Netw., vol. 18, pp. 851-864, 2007.

[4] A. Abraham, A. Yeung, "Integrating ensemble of intelligence systems for stock exchange prediction," Notes on Computational Science, vol. 2687, pp. 774-781, 2003.

[5] W. Härdle, W. Lee, D. Schäfer, Y. Yeh, "Variable selection and oversampling in the use of smooth support vector machines for predicting the default risk of companies," Journal of Forecasting, vol. 28, pp. 512-534, 2009.

[6] N. Sapankevych, R. Sangar, "Time series prediction using support vector machines: a survey," IEEE Computational Intelligence Magazine, vol.4, pp. 24-38, 2009.

[7] A. Khandani, A. Kim, A. Lo, "Consumer credit-risk models via machine-learning algorithms," J. Bank. Finance, vol. 34, pp. 2767-2787, 2010

[8] H. Öğüt, M. Doğanay, N. Ceylan, R. Aktaş, "Prediction of bank financial strength ratings: the case of Turkey," Economic Modelling, vol. 29, pp. 632-640, 2012.

[9] T. Papadimitriou, P. Gogas, M. Matthaiou, E. Chrysanthidou, "Yield curve and recession forecasting in a machine learning framework," Computational Economics, vol. 45, pp. 635-645, 2015.

[10] M. Lam, "Neural network techniques for financial performance prediction: integrating fundamental and technical analysis," Decis. Support Syst., vol. 37, pp. 567-581, 2004.

[11] C. Dunis, M. Williams, "Modeling and trading the EUR/USD exchange rate: do neural network models perform better?," Derivatives Use, Trading and Reg., vol. 8, pp. 211-239, 2002.

[12] R. K. Bissoondeeal, M. Karoglou, A.M. Gazely, "Forecasting the UK/US exchange rate with divisia monetary models and neural networks," Scott. J. Political Econ., vol. 58, pp. 127-152, 2011.

[13] E. Önder, B. Firat, A. Hepșen, "Forecasting macroeconomic variables using artificial neural network and traditional smoothing techniques," J. Appl. Finance Bank., vol. 3, pp. 73-104, 2013..

[14] H. Ince, T. Trafalis, "A hybrid model for exchange rate prediction," Decision Support Systems, vol. 42, pp. 1054-1062, 2006.

[15] B. Brandl, U. Wildburger, S. Pickl, "Increasing of the fitness of fundamental exchange rate forecast models," International Journal of Contemporary Mathematical Sciences, vol. 4, pp. 779-798, 2009.

[16] G. Rubio, H. Pomares, I. Rojas, L. Herrera, "A heuristic method for parameter selection in LS-SVM: application to time series prediction," International Journal of Forecasting, vol. 27, pp. 725-739, 2011.

[17] K. Chen, C. Wang, "Support vector regression with genetic algorithms in forecasting tourism demand," Tourism Management, vol. 28, pp. 215-226, 2007.

[18] G. Sermpinis, C. Stasinakis, K. Theofilatos, A. Karathanasopoulos, "Modeling, forecasting and trading the EUR exchange rates with hybrid rolling genetical gorithms-Support vector regression forecast combinations," Euro. J. Oper. Res., vol. 247, pp. 831-846, 2015 